\title{
Broadband Terahertz Time-Domain Spectroscopy of Archaeological Baltic Amber
}

\author{
Toru Sasaki ${ }^{1}$, Yusuke Hashimoto ${ }^{2}$, Tatsuya Mori ${ }^{2, a}{ }^{*}$, Seiji Kojima ${ }^{2}$ \\ ${ }^{1}$ School of Engineering Sciences, University of Tsukuba, Tsukuba, Ibaraki 305-8573, Japan \\ ${ }^{2}$ Graduate School of Pure and Applied Sciences, University of Tsukuba, \\ Tsukuba, Ibaraki 305-8573, Japan \\ amori@ims.tsukuba.ac.jp
}

Keywords: amber, terahertz time-domain spectroscopy, dielectric constant, boson peak

\begin{abstract}
We performed the broadband terahertz time-domain spectroscopy on archaeological Baltic amber at 40 million years old. We determined the real and imaginary parts of the complex dielectric constants in the frequency range from 0.2 to $6.5 \mathrm{THz}$. The imaginary part of the complex dielectric constants showed a broad peak at $1.51 \mathrm{THz}$ and a small peak at $4.0 \mathrm{THz}$. A boson peak, which is the characteristic low energy excitation of a glassy state, was not observed above $0.2 \mathrm{THz}$ even in low temperatures.
\end{abstract}

\section{Introduction}

A fossilized resin is called amber, which has spent maturation processes over geological time. Amber is a unique example of natural glasses for various kinds of researches, because they have aged for quite a long time below its glass transition temperature [1]. The glass transition temperature $T_{g}$ of amber doesn't show a large variation, and the $T_{g}$ of amber shows a distribution between about $390 \mathrm{~K}$ and $460 \mathrm{~K}$ [2].

One of the universal features of glassy materials is the low-energy state called a boson peak [3]. The boson peak of the 110 million-year-old amber from El Soplao (Spain) was investigated by the specific heat, $C_{p}$, at low temperatures, and the peak of $C_{p} / T^{3}$ was observed at about $3 \mathrm{~K}$ [1]. Therefore, the boson peak frequency of this amber in dynamical properties is expected to be about $0.3 \mathrm{THz}$.

THz-TDS has been adequately recognized to be one of trustworthy tools for spectroscopic measurements of far-infrared properties of materials like dielectrics $[4,5]$, semiconductors $[4,6]$, superconductors [7], gases [8], liquids [8] and glassy materials [9,10]. In the present study, we performed broadband terahertz time-domain spectroscopy to investigate the dynamical properties of Baltic amber in the $\mathrm{THz}$ range. Baltic amber is originated from a vast 'amber forest' in Scandinavia about 60 million years ago [11].

\section{Experimental}

The archaeological ambers we measured were obtained in Baltic Sea, and the age of the ambers is about 40 million years old. We cut and polished the ambers in order to form for THz-TDS measurements, and the thicknesses of the samples for THz-TDS measurement were 2.311 and 0.912 $\mathrm{mm}$ to obtain enough intensity of transmission spectra.

The present THz-TDS measurements were performed by using the conventional transmission configuration (RT-10000, Tochigi Nikon Corp.) and another THz-TDS system (TAS7500SU, Advantest Corp.). The broad frequency range is from 0.2 to $6.5 \mathrm{THz}$ by using the two different THz-TDS systems. The low-frequency $\mathrm{THz}$ transmission spectra were measured in the frequency from 0.2 to $4.0 \mathrm{THz}$ by RT-10000, and the broadband THz transmission spectra were measured from 0.5 to $6.5 \mathrm{THz}$ by TAS7500SU. In the RT-10000, Low-temperature grown GaAs photoconductive (PC) antennas were utilized for the $\mathrm{THz}$ pulse emitter and detector, and the PC antennas were triggered by a mode-locked Ti:sapphire pulsed laser with a wave-length of $780 \mathrm{~nm}$, a pulse width of 
less than $100 \mathrm{fs}$, and a repetition rate of $80 \mathrm{MHz}$. The ultrafast THz-TDS, the TAS7500SU utilized a high-speed asynchronous optical sampling technique in which two femtosecond lasers were used instead of the mechanical delay stage [9]. In TAS7500SU, $\mathrm{MgO}$ doped $\mathrm{LiNbO}_{3}$ (Cherenkov phase matching) and photoconductive antenna were utilized for the $\mathrm{THz}$ pulse emitter and detector respectively, and they were triggered by a femtosecond fiber laser with a wave-length of $1560 \mathrm{~nm}$, a pulse width of under $45 \mathrm{fs}$, and a repetition rate of $50 \mathrm{MHz}$. In data analysis, we smoothly connected the absolute value of the complex dielectric constants of the same sample with different thickness as based on of the low frequency data of the thin sample at $2.4 \mathrm{THz}$ [9]. The temperature of the sample measured by RT-10000 was controlled by the liquid helium flowing within the range from $300 \mathrm{~K}$ to $10 \mathrm{~K}$ in cooling and $10 \mathrm{~K}$ to $310 \mathrm{~K}$ in heating process, and the sample for TAS7500SU was measured at room temperature $(296 \mathrm{~K})$.

Figure 1 (a) shows the measured time-domain $\mathrm{THz}$ E-field waveforms transmitted through the air (reference) and the amber whose thickness is $2.311 \mathrm{~mm}$. Figure 1(b) shows the frequency-domain power spectra. To calculate the frequency dependent complex dielectric constant $\hat{\varepsilon}=\varepsilon^{\prime}+i \cdot \varepsilon^{\prime \prime}$ from the measured time-domain waveforms, we have used following equation,

$$
\frac{E_{\text {sam }}(v)}{E_{\text {ref }}(v)}=\frac{4 \hat{n}}{(\hat{n}+1)^{2}} \exp \left\{i \frac{(\hat{n}-1)}{c} \omega d\right\},
$$

where $E_{\text {sam }}(v)$ and $E_{\text {ref }}(v)$ are the amplitude spectra of $\mathrm{THz}$ pulse transmitted the sample and the reference, respectively. $\hat{n}, c$ and $d$ are the complex refractive index, the light velocity and a thickness of sample, respectively. The complex dielectric constant is obtained from the relation of $\hat{\varepsilon}=\hat{n}^{2}$ [12].
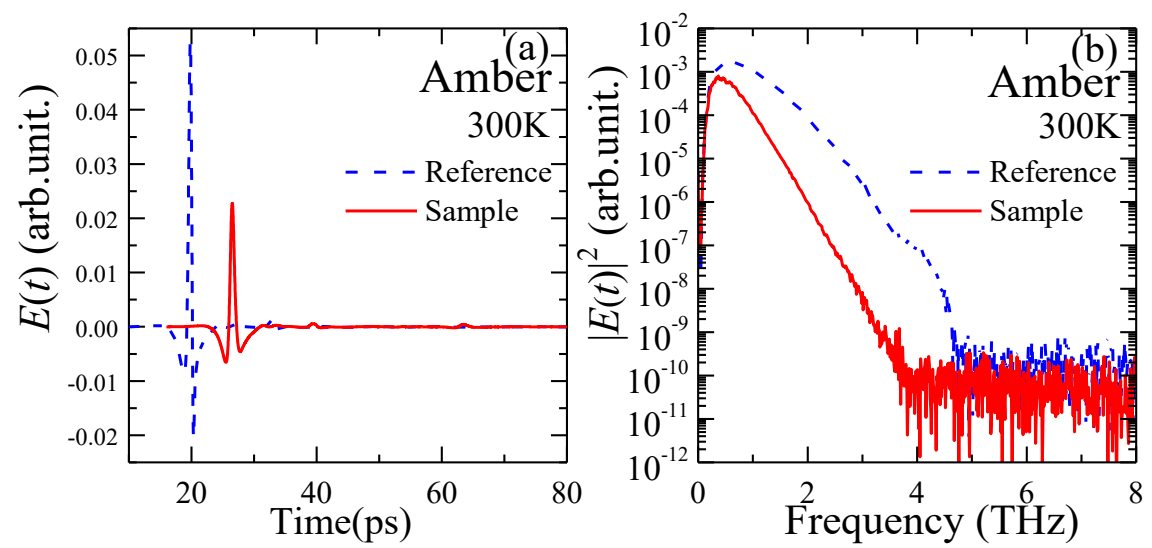

Figure 1. (a) Time-domain waveforms and (b) frequency-domain power spectra of the air (reference) and the amber at $300 \mathrm{~K}$ by the RT-10000.
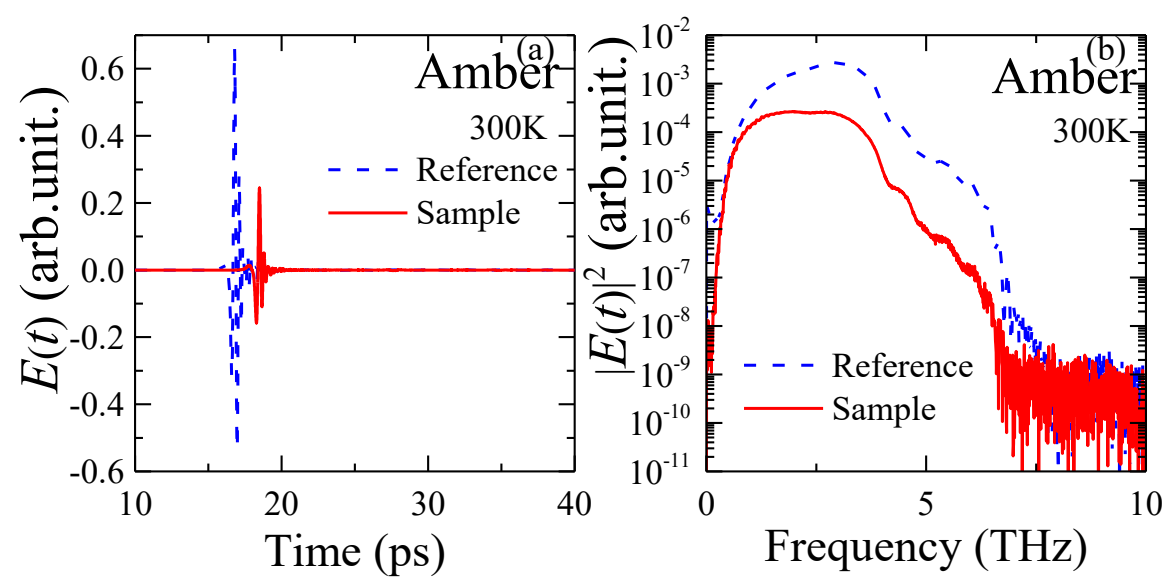

Figure 2. (a) Time-domain waveforms and (b) frequency-domain power spectra of the air (reference) and the amber at $300 \mathrm{~K}$ by the TAS7500SU. 
Figure 2 (a) shows the measured time-domain $\mathrm{THz} E$-field waveforms transmitted through the air (reference) and the amber whose thickness is $0.912 \mathrm{~mm}$. Figure 2(b) shows the frequency-domain power spectra.

\section{Results and Discussion}

Figures 3(a) and 3(b) show the real and imaginary parts of the complex dielectric constants of the amber. The real part of the dielectric constant, $\varepsilon^{\prime}(v)$ shows a decrease toward high temperatures, while the imaginary part of the dielectric constant, $\varepsilon^{\prime \prime}(v)$ shows a broad peak at around $1.5 \mathrm{THz}$ and a small peak at around $4.0 \mathrm{THz}$.

This frequency range is dominated by universal feature of glasses, so-called boson peak arising from an excess in the vibrational density of state (VDOS) $[1,3,13,14]$. The imaginary parts of the complex dielectric constant are related to VDOS $g(v)$ through infrared (IR) coupling constant $C_{I R}(v)$, and the relation is expressed by using following equation [3],

$$
\frac{2 \pi}{c \cdot n^{\prime}(v)} \cdot \frac{\varepsilon^{\prime \prime}(v)}{v}=C_{I R}(v) \cdot \frac{g(v)}{v^{2}},
$$

where $n^{\prime}(v)$ and $C_{I R}(v)$ are the real part of refractive index and IR coupling constant, respectively. Therefore we can find the boson peak from the spectrum of $\varepsilon^{\prime \prime}(v) /\left(n^{\prime} \cdot v\right)$. According to specific heat investigation [1], the boson peak of ambers should be observed $t$ at around $0.3 \mathrm{THz}$.

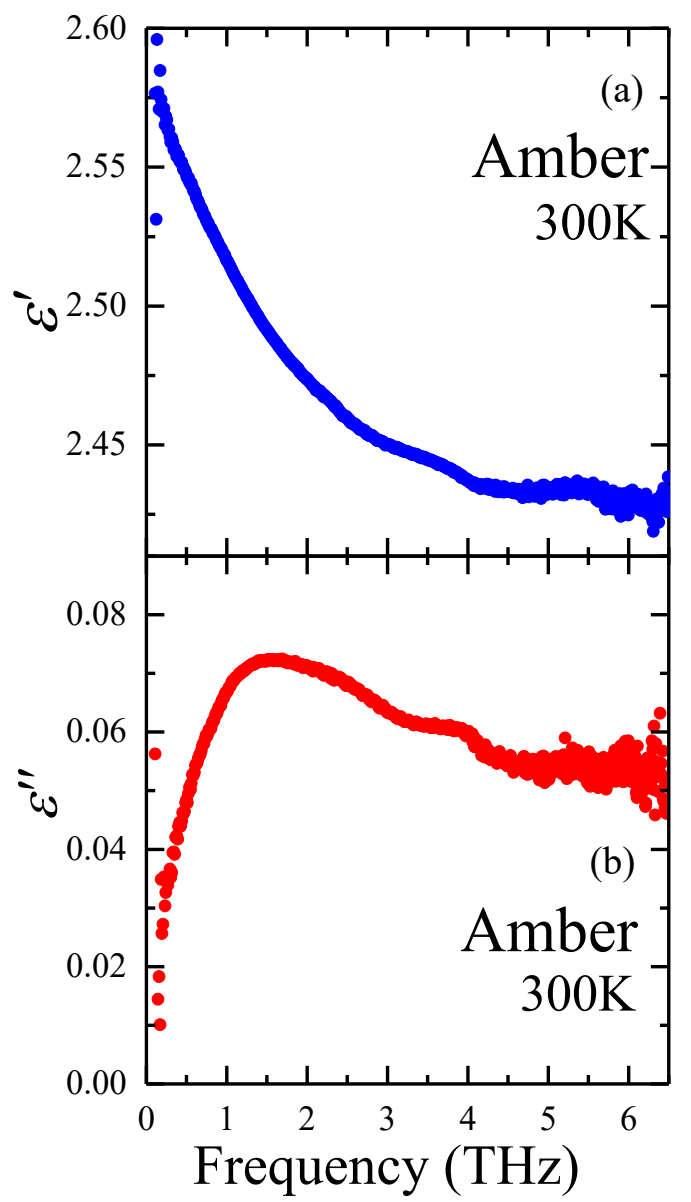

Figure 3. (a) The real part $\varepsilon^{\prime}(v)$ and (b) the imaginary part of the complex dielectric constant $\varepsilon^{\prime \prime}(v)$ of the amber at $300 \mathrm{~K}$. 


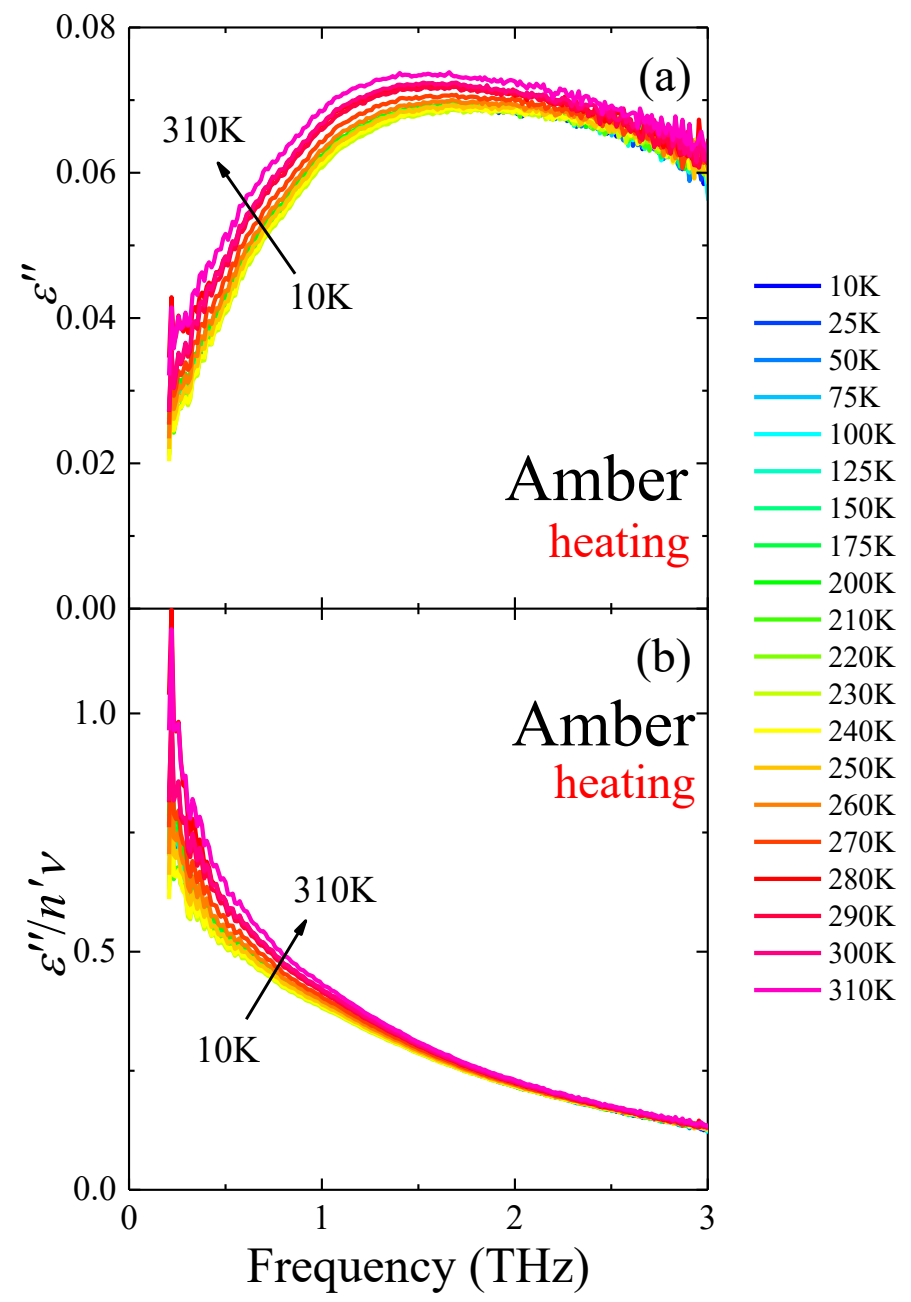

Figure 4. (a) The imaginary part of the complex dielectric constants at each temperature and (b) the imaginary part divided by $n^{\prime} \cdot v$ of the amber at each temperature.

To observe the temperature dependence of the amber, we changed the temperature of the sample within the range from $300 \mathrm{~K}$ to $10 \mathrm{~K}$ in cooling process and $10 \mathrm{~K}$ to $310 \mathrm{~K}$ in heating process. Figure 4 (a) shows a similarity of the spectral shapes of the imaginary part of dielectric constants upon heating processes. The imaginary part of the complex dielectric constant divided by $n^{\prime} \cdot v$ of the amber is shown in Fig. 4 (b). However, boson peak was not observed in IR spectra even in low temperatures. According to the study of molecular dynamical simulation by Shintani and Tanaka shows that the boson peak frequency is proportional to the shear modulus [15]. Such a correlation was experimentally confirmed in lithium borate glasses [16]. The possible reason on the absence of a boson peak above $0.2 \mathrm{THz}$ is that the boson peak frequency is less than $0.2 \mathrm{THz}$ by the soft structure of relatively young amber. The shear modulus of younger amber is smaller than that of the old one reported in a previous report of specific heat investigation [1], more than 10 million years while the amber we measured is 4 million years old. Figure 5 shows the temperature dependence of imaginary parts of the complex dielectric constants of the amber at $1.5 \mathrm{THz}$. The values of imaginary part of complex dielectric constants didn't show temperature dependence between $10 \mathrm{~K}$ and around $200 \mathrm{~K}$. However, it increased above around $200 \mathrm{~K}$ toward high temperature, and it showed small thermal hysteresis at around $200 \mathrm{~K}$. The change of the tendency of the imaginary part at around $200 \mathrm{~K}$ can be attributed to the existence of water in very small spaces of amber. Amber usually contains water and loses it gradually with passage of time. There is a possibility that the solid-liquid phase transition of confined water in very small spaces by nucleation doesn't happen and the water becomes into supercooled state and glass transition at around $200 \mathrm{~K}$ finally happens. 


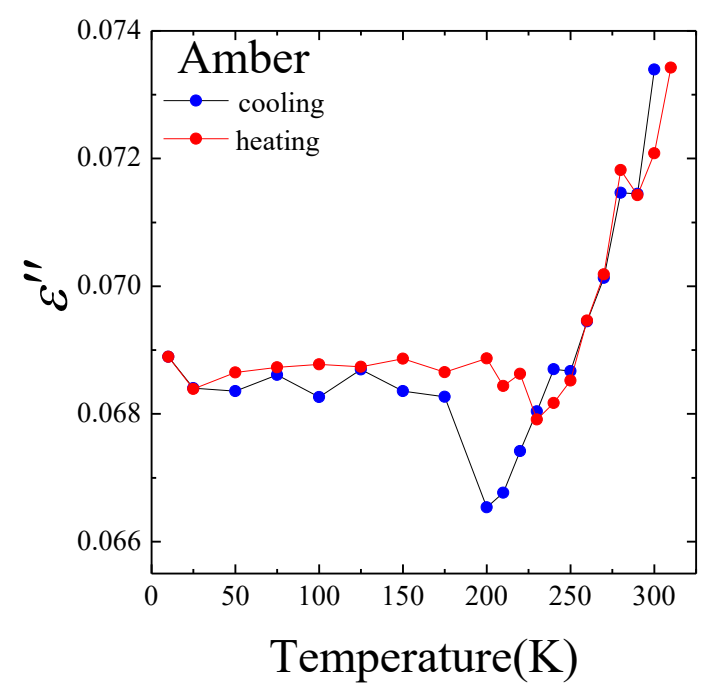

Figure 5. The temperature dependence of imaginary parts of the complex dielectric constants of the amber at $1.5 \mathrm{THz}$.

\section{Conclusions}

The THz dynamics of the archaeological Baltic amber at 40 million years old was investigated by using broadband THz-TDS. The boson peak wasn't observed above $0.2 \mathrm{THz}$ in the IR spectra $\varepsilon^{\prime \prime}$ $(v) / n^{\prime} \cdot v$ even at low temperatures. It can be attributed to the smaller modulus originated from younger age of the present amber. The change of the tendency of $\varepsilon^{\prime \prime}(v)$ at around $200 \mathrm{~K}$ can be caused by the liquid-glass transition of confined waters in voids of amber.

\section{Acknowledgements}

The authors are very thankful for the technical support of the broadband THz-TDS measurements by the Advantest Corporation. This study was partially supported by a Grant-in-Aid for Young Scientists (B) (24740194) from JSPS and Casio Science Promotion Foundation.

\section{References}

[1] T. Perez-Castaneda, R. J. Jimenez-Rioboo, M. A. Romas, Phys. Rev. Lett. 112, (2014) 165901.

[2] J. Zhao, G B. McKenna, Poly. 55 (2014) 2246-2253.

[3] F. L. Galeener, and P. N. Sen, Phys. Rev. B 17 (1978) 1928.

[4] D. Grischkowsky, S. Keiding, M. van Exter, and C. Fattinger, J. Opt. Soc. Am. B 7 (1990) 2006.

[5] H. Igawa, T. Mori, and S. Kojima, Jpn. J. Appl. Phys. 53 (2014) 05FE01.

[6] T.Mori, K. Iwamoto, S. Kushibiki, H. Honda, H. Matsumoto, and N. Toyota, Phys. Rev. Lett. 106 (2011) 015501.

[7] T. Mori, E. J. Nicol, S. Shiizuka, K. Kuniyasu, T. Nojima, N. Toyota, and J. P. Carbotte, Phys. Rev. B 77 (2008) 174515.

[8] B. B. Hu and M. C. Nuss, Opti, Lett. 20 (1995) 16.

[9] T. Shibata, T. Mori, and S. Kojima, Spectrochim. Acta A: Mol. Biomol. Spectrosc. 150 (2015) 207.

[10]S. Kojima, T. Mori, T. Shibata and Y. Kobayashi, Pharm Anal Acta. 6 (2015) 8.

[11]Y. Shashoua, M. B. L. D. Berthelsen, O. F. Nielsen, J. Raman Spectrosc. 37 (2006) 1221-1227.

[12]Y. Kobayashi, T. Shibata, T. Mori, S. Kojima, Inte. lett. Chem. Phys. Astr, 46 (2015) 16-22.

[13]M. Foret, E. Courtens, R. Vacher, J.-B. Suck, Phys. Rev. Lett. 77 (1996) 3831.

[14]T. Nakayama, Rep. Prog. Phys. 65 (2002) 1195.

[15]H. Shintani, H. Tanaka, Nat. Mater. 7 (2008) 870.

[16]S. Kojima, Y. Matsuda, Y. Fukawa, M. Kawashima, Y. Moriya, T. Yamada, O. Yamamuro, M. Kodama, J. Non-Crys. Solids, 356 (2010) 2524. 